\title{
The structure of fadL mRNA and its interactions with RybB sRNA
}

\author{
Agata Groszewska, Zuzanna Wroblewska and Mikolaj Olejniczak ${ }^{\bowtie}$ \\ Department of Biochemistry, Institute of Molecular Biology and Biotechnology, Faculty of Biology, Adam Mickiewicz University, Poznań, Poland
}

Small bacterial RNAs (sRNAs) regulate translation by pairing with complementary sequences in their target mRNAs, in a process which is often dependent on the Hfq protein. Here, the secondary structure of a 95-nt long fragment of Salmonella fadL mRNA containing RybB sRNA binding site in the coding region was analyzed. The data indicated local rearrangements in this mRNA structure after the annealing of RybB. The filter retention data had shown that Hfq bound both RybB and the fadL mRNA fragment with tight affinities. Moreover, $\mathrm{Hfq}$ increased the rate of RybB annealing to fadL mRNA. These data indicate that Hfq directly participates in RybB interactions with the fadL mRNA.

Key words: Hfq, sRNA, mRNA, RybB, fadL, coding sequence

Received: 06 June, 2016; revised: 21 October, 2016; accepted: 24 October, 2016; available on-line: 25 November, 2016

\section{INTRODUCTION}

Bacterial trans-encoded small noncoding RNAs (sRNAs) participate in the cell's adaptation to changing environmental conditions and in the maintance of cellular homeostasis (Waters \& Storz, 2009). They are also involved in the regulation of bacterial pathogenicity (Papenfort \& Vogel, 2010; Tree et al., 2014). Small RNAs control gene expression by pairing with complementary sequences in the regulated mRNAs, which affects mRNA translation and decay (Wagner \& Romby, 2015). In particular, small RNAs regulate the translation of the outer membrane proteins in Escherichia coli and Salmonella Typhimurium, which include the OmpC (Chen et al., 2004), OmpD (Pfeiffer et al., 2009), and OmpN porins (Bouvier et al., 2008). The sRNA-dependent regulation of the outer membrane composition affects such important processes as the uptake of nutrients (FigueroaBossi et al., 2009), response to membrane stress (Guo et al., 2014), biofilm formation (Jorgensen et al., 2013), and the resistance to antibiotics (Parker \& Gottesman, 2016).

Translation regulation by many of sRNAs is dependent on the ring-shaped Hfq protein (Updegrove et al., 2016). Hfq contains three RNA binding sites on its surface, which allows it to use different binding modes in interactions with the sRNA and mRNA molecules (Zhang et al., 2013; Schu et al., 2015). The positively charged residues on the rim of the $\mathrm{Hfq}$ ring are necessary for efficient annealing of the sRNA and mRNA molecules (Panja et al., 2013; Zheng et al., 2016), while the negatively charged residues (Panja et al., 2015), and the C-terminal protein extensions (Santiago-Frangos et al., 2016) contribute to the specificity of the interactions. In the best studied interaction of $E$. coli DsrA sRNA with the rpoS mRNA, the role of $\mathrm{Hfq}$ is to rearrange the
mRNA structure to facilitate the sRNA annealing (Soper \& Woodson, 2008; Soper et al., 2011; Peng et al., 2014a; Peng et al., 2014b). A recent study of three sRNAs binding to Salmonella ompD mRNA had shown that the role of Hfq in rearranging sRNA and mRNA structures depends on the structural properties of the interacting RNAs (Wroblewska \& Olejniczak, 2016b).

RybB is an Hfq-dependent sRNA that represses the synthesis of several outer membrane proteins in response to the envelope stress (Balbontin et al., 2010; Papenfort et al., 2010). The 5'-end of this 79-nt long sRNA functions as an autonomic regulatory domain, which is essential for sRNA binding to different mRNA targets. Among targets of RybB is the fadL mRNA, which encodes an outer membrane porin involved in the transport of long-chain fatty acids (Nunn \& Simons, 1978; Black et al., 1987). RybB sRNA represses its translation by binding downstream of the AUG start codon, at positions +43 to +50 of the coding sequence (Papenfort et al., 2010).

To better elucidate the interactions between RybB sRNA and fadL mRNA, changes in the structure of a 95-nt long fadL mRNA fragment upon RybB binding were monitored. Moreover, the $\mathrm{Hfq}$ protein binding to both RNAs and its influence on the kinetics of RybB annealing to the coding sequence of fadL were studied.

\section{MATERIALS AND METHODS}

RNA preparation. DNA templates for in vitro transcription were obtained by Taq polymerase extension of chemically synthesized, overlapping oligonucleotides (oligo.pl, Warsaw, Poland). Salmonella RybB sRNA and fadL95 mRNA fragment were in vitro transcribed using T7 RNA polymerase and purified using denaturing PAGE as described (Milligan et al., 1987). RNA molecules were 5'-32P-labeled using T4 polynucleotide kinase (Thermo Scientific) and purified on denaturing PAGE. After elution, the samples were ethanol precipitated, dried, and dissolved in water to a final $200 \mathrm{nM}$ RNA concentration.

Hfq protein purification. The Salmonella C-terminally $\mathrm{His}_{6}$-tagged $\mathrm{Hfq}$ protein was overexpressed and purified as previously described for E. coli Hfq (Malecka et al., 2015). In short, after overexpression from pET15b plasmid, the cells were lysed by sonication, and the protein was purified by $\mathrm{Ni}^{2+}$ affinity chromatography (HisTrap crude column, GE Healthcare), followed by treatment with DNase I and RNase A to remove any bound nu-

e-mail: mol@amu.edu.pl

Abbreviations: ANN, a repeated sequence in which every third nucleotide is $A ; \mathrm{Hfq}$, host factor for phage $\mathrm{Q}$ beta replication; $\mathrm{Kd}$, equilibrium dissociation constant; kobs, observed association rate constant; sRNA, small noncoding RNA 
cleic acids. Next, the size exclusion purification was performed on a HiLoad 16/60 Superdex 200 size exclusion column (GE Healthcare) equilibrated with storage buffer (50 mM HEPES 7.5, $250 \mathrm{mM} \mathrm{NH} \mathrm{NH}_{4}, 1 \mathrm{mM}$ EDTA, and $10 \%$ glycerol). The $\mathrm{Hfq}$ concentration was determined using absorbance at $280 \mathrm{~nm}$ (Olejniczak, 2011).

In vitro structure probing and footprinting. Just before setting up the reactions, the ${ }^{32} \mathrm{P}$-fadL95 RNA was denatured at $90^{\circ} \mathrm{C}$ for $1 \mathrm{~min}$ and then incubated at room temperature for $10 \mathrm{~min}$. The structure probing experiments were performed essentially as previously described (Wroblewska \& Olejniczak, 2016b). The reactions with RNase T1 were carried out in $12 \mathrm{mM}$ Tris- $\mathrm{HCl}, \mathrm{pH}$ 7.2, $48 \mathrm{mM} \mathrm{NaCl}$, and $1.2 \mathrm{mM} \mathrm{MgCl}_{2}$ at RT for 10 min. The RNase $\mathrm{T} 2$ reactions were performed in 10

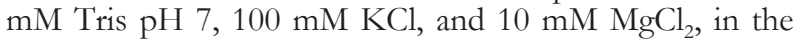
presence of $1 \mu \mathrm{g}$ of yeast tRNA, and incubated at RT for $15 \mathrm{~min}$. Structure probing reactions with nuclease $\mathrm{S} 1$ were performed in $40 \mathrm{mM}$ sodium acetate $\mathrm{pH} 4.5$,
A
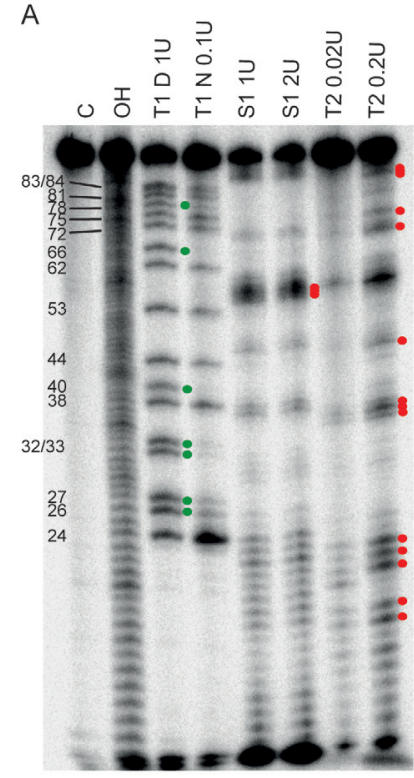

C

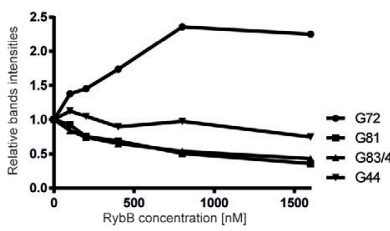

B

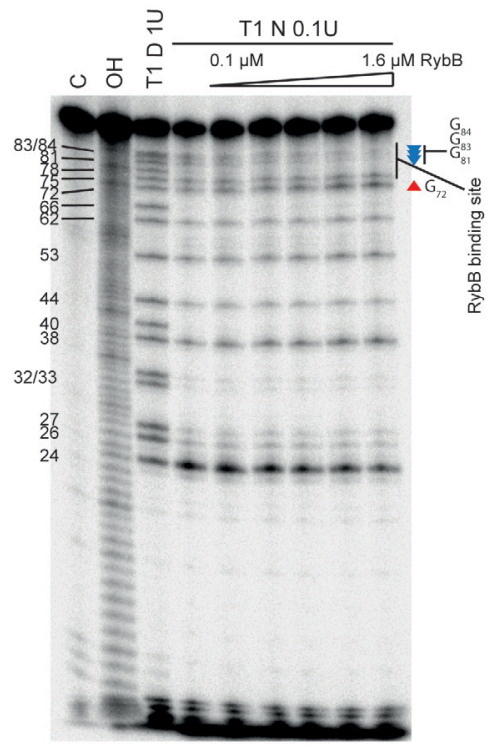

$\mathrm{D}$

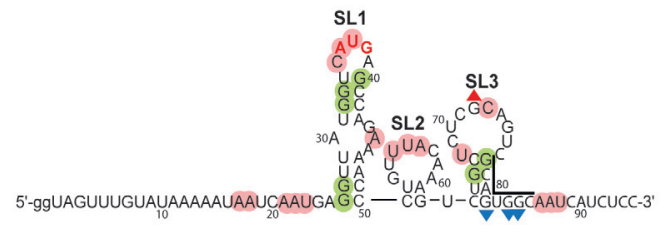

Figure 1. The annealing of RybB SRNA induces changes in the secondary structure of the fadL95 mRNA fragment.

(A) In vitro structure probing of ${ }^{32} \mathrm{P}$-fadL mRNA fragment was performed using RNase T1 and T2, and the S1 nuclease (indicated above the lanes). (B) The RNase T1 structure probing of ${ }^{32} \mathrm{P}$-fadL mRNA fragment in the presence of increasing concentration of RybB. The guanosine residue more susceptible to RNase T1-induced cleavages is indicated by a red triangle, while the residues less susceptible to this cleavage are indicated by blue reverse triangles. RNase $T 1$ was used under native (T1 N) and denaturing (T1 D) conditions, $C$ is an untreated control, while $\mathrm{OH}$ indicates the formamide ladder. Numbers on the left side of the gel correspond to the $\mathrm{G}$ residue positions. (C) Changes in nucleotide susceptibility to cleavage upon RybB binding were plotted versus RybB concentration. (D) Secondary structure of the fadL95 mRNA based on the structure probing results presented in (A). Residues indicated in red and green were constrained as single- or double-stranded, respectively, to generate the structure model using the RNAstructure program. The region complementary to RybB sRNA is marked by lines, AUG start codon is indicated by red font. The position of increased cleavage with RNase T1 upon RybB binding is indicated by a red triangle, and the positions of decreased cleavage with blue reverse triangles.
$300 \mathrm{mM} \mathrm{NaCl}$, and $2 \mathrm{mM} \mathrm{ZnSO}_{4}$, and incubated at RT for 10 min. Reactions with RNase T1, T2 and S1 were and $20 \mathrm{mM}$ adition of $10 \mu \mathrm{L}$ of stop buffer ( $\mathrm{M}$ urea above. Samples were separated on $10 \%$ polyacrylamide

Equilibrium binding assays. The affinity of $\mathrm{Hfq}$ to ${ }^{32}$ P-labeled RybB and fadL95 molecules was measured using a high-throughput double filter retention assay as described (Olejniczak, 2011). The binding reactions contained $24 \mathrm{mM}$ Tris- $\mathrm{HCl}(\mathrm{pH}$ 7.5), $50 \mathrm{mM} \mathrm{NH} \mathrm{ml}_{4}, 50 \mathrm{mM} \mathrm{NaCl}$, $50 \mathrm{mM} \mathrm{KCl}, 5 \%$ glycerol, and 0.5 $\mathrm{mM}$ EDTA (Lease \& Woodson, 2004). Prior to use, ${ }^{32}$ P-labeled RNA molecules were denatured for $2 \mathrm{~min}$ at $90^{\circ} \mathrm{C}$ followed by incubation at room temperature for $10 \mathrm{~min}$. The binding reactions were initiated by mixing $15 \mu \mathrm{L}$ of 5 '-32Plabeled RNA at $0.02 \mathrm{nM}$ concentration with $15 \mu \mathrm{L}$ of $\mathrm{Hfq}$ dilution followed by incubation for $1 \mathrm{~h}$ at RT. $25 \mu \mathrm{L}$ aliquots of each reaction were withdrawn, filtered and washed with $100 \mu \mathrm{L}$ of binding buffer. Filters were dried, exposed to phosphor screens, and the data were quantified using a phosphorimager and MultiGauge software. The binding data were fit to the Michaelis-Menten binding isotherm.

To monitor the equlibrium binding of ${ }^{32}$ P-labeled fadL95 to RybB sRNA, an electrophoretic mobility shift assay was used. Prior to use, RNAs were treated as described above. The equilibrium binding reactions were prepared by mixing $15 \mu \mathrm{L}$ of ${ }^{32} \mathrm{P}$-labeled fadL95 at 1 $\mathrm{nM}$ final concentration with $15 \mu \mathrm{L}$ of RybB solution followed by incubation for $1 \mathrm{~h}$ at RT. $5 \mu \mathrm{l}$ of each sample was loaded on a $6 \%$ native polyacrylamide gel in $1 \times$ TBE buffer. The gels were run at $5 \mathrm{~W}$ at $4^{\circ} \mathrm{C}$ in a coldroom, dried, and analyzed using a phosphorimager. The fraction bound data were fit to the Michaelis-Menten binding isotherm.

Annealing assays. The kinetics of annealing of RybB sRNA to fadL95 mRNA fragment in the presence or absence of Hfq protein was monitored by gel mobil- 
ity shift assay as described (Wroblewska \& Olejniczak, 2016b) with the following modifications. The annealing reactions were prepared in $80 \mu \mathrm{L}$ total volume by mixing $1 \mathrm{nM}{ }^{32} \mathrm{P}$-fadL95 with $50 \mathrm{nM}$ RybB sRNA in the presence or absence of $3 \mathrm{nM} \mathrm{Hfq}$ (hexamer concentration). The reactions were incubated at $25^{\circ} \mathrm{C}$ in a thermomixer. $5 \mu \mathrm{L}$ aliquots were withdrawn at indicated time points and separated on $6 \%$ native polyacrylamide gels in $1 \times$ TBE buffer at $4^{\circ} \mathrm{C}$ in a coldroom. Control reactions were prepared in the same way, except that to the reactions with ${ }^{32} \mathrm{P}$-fadL95 and Hfq, the unlabeled fadL95 RNA at $2 \mathrm{nM}$ concentration was added to prevent the precipitation of the sample in the wells. The gels were run continuously during experiments. After quantifying the data using a phosphorimager, the fraction bound values of RybB-fadL95 or RybB-fadL95-Hfq complexes were plotted versus time (0.5-60 minutes). Observed association rates $\left(\mathrm{k}_{\mathrm{obs}}\right)$ were determined by fitting the data to the single exponential equation.

\section{RESULTS}

RybB sRNA binds fadL mRNA at the sequence which is localized +43 to $+50 \mathrm{nt}$ from the translation start site (Papenfort et al., 2010). To analyze RybB interactions with fadL, a 95-nt long fragment of this mRNA was used (fadL95) (Fig. 1D). It included a 35-nt long sequence of 5 '-untranslated region (5'-UTR) of this mRNA and a 60 -nt long region of its coding sequence, including the RybB binding site. This fragment contained a 16-nt long AU-rich sequence in the 5'-untranslated region, which could be described as (ANN), repeated sequence. Similar sequences were shown as functional $\mathrm{Hfq}$ binding sites in other mRNA molecules (Soper \& Woodson, 2008; Schu et al., 2015; Wroblewska \& Olejniczak, 2016b). It also included a shorter 8-nt long AU-rich sequence in the coding region (Fig. 1D). As a control, a longer, 216-nt fragment including the whole 100-nt 5'-UTR of fadL mRNA was also tested; however, its multiple complexes with Hfq could not be well separated on the gel (data not shown). Hence, the fadL95 mRNA fragment was used in this study, which contained both the region of RybB binding and a lengthy AU-rich sequence in the 5'-UTR.

To determine the secondary structure of fadL95 mRNA the probing with RNase T2 and nuclease S1 was applied to detect single-stranded regions, while the comparison of cleavages generated by RNase T1 under the denaturing and native conditions was used to define double-stranded regions. The structural information provided by enzymatic probing was used to predict the secondary structure of fadL95 using the RNAstructure software (Reuter \& Mathews, 2010). The most thermodynamically stable of the two structures predicted by the software based on the experimental constraints is presented in Fig. 1D.

The secondary structure of the region of fadL mRNA surrounding the translation start site contained three stem-loop structures, named SL1 to SL3 (Fig. 1A, D). The double-stranded character of SL1 and SL3 was supported by decreased RNase T1-induced cleavage under native conditions of the SL1 G26, G27, G32, G33, and G40 residues, and the SL3 G66 and G78 residues. The fact that guanosine residues at positions 83 and 84 were quite accessible to RNase T1 under native conditions, as well as the A86 - U88 residues to RNases S1 and T2, suggested that they form an elongated single-stranded region. Residue A45, susceptible to RNase T2, is located within the 3' part of the internal asymmetric loop of
SL1. Additionally, cleavages induced by RNase T2 and nuclease S1 at positions U55-A57 defined the apical loop of SL2, while U68 and C73 the apical loop of SL3 (Fig. 1A, D).

Both AU-rich sequences in fadL95 mRNA are located in conformationally dynamic regions, while the RybB binding site partially overlaps with a stem-loop structure (Fig. 1A, D). The long AU-rich sequence in the 5'-UTR is composed of unpaired nucleotides and it could be accessible for binding by the Hfq protein (Fig. 1A, D). The short AU-rich sequence in the coding region is also single-stranded and forms a loop of SL2. The GAGG Shine-Dalgarno sequence is partly involved in the formation of the SL1, which could limit its availability to the ribosome, while the AUG start codon is located in the apical loop of SL1. The predicted binding site of RybB sRNA is located within the stem of SL3 structure and in the following single-stranded region (Fig. 1A, D).

Annealing of RybB sRNA to fadL95 induced changes in the structure probing pattern in the area of its binding site, which is consistent with the unfolding of SL3 (Fig. 1B, C). The G81 residue in the SL3, as well as G83 and G84 in the following single-stranded region, which are part of the sequence complementary to the 5'-terminus of RybB sRNA, were increasingly protected from RNase T1 induced cleavage with the increase of RybB concentration (Fig. 1B, C). As a control, the cleavage intensities of a distantly located G44 residue were also compared, and have shown only a small change upon RybB annealing. On the other hand, the G72 residue, which is located in the apical loop of SL3, has displayed an increased susceptibility to RNase T1-induced degradation, when RybB concentration was increased (Fig 1B, C, D). These data are consistent with the unfolding of the SL3 stem-loop upon RybB binding and improved accessibility of residues located within the SL3
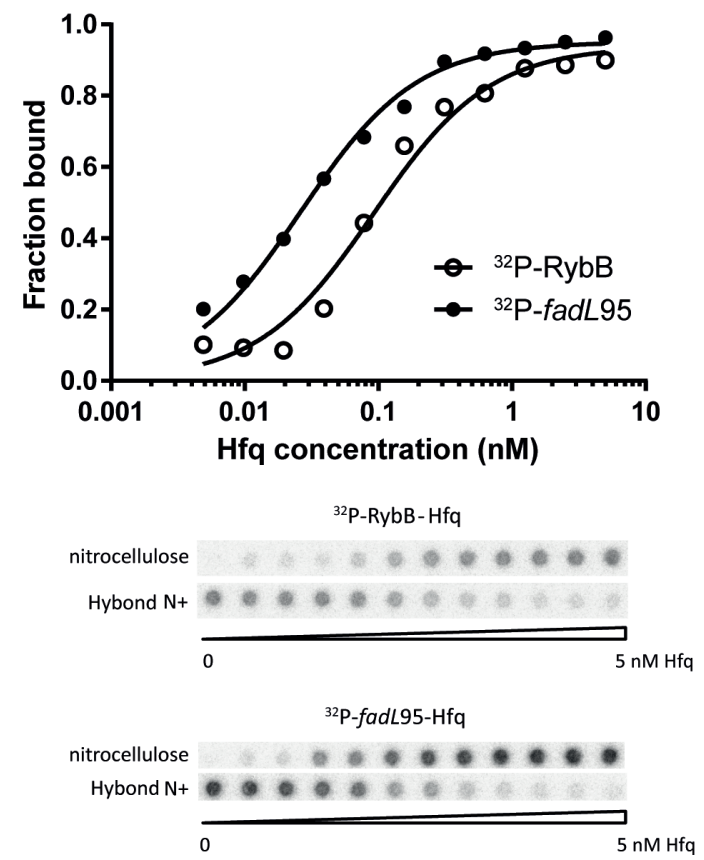

Figure 2. The binding of the RybB sRNA and fadL95 mRNA to the Hfq protein.

The bound fraction data provided by filter retention assay (raw data shown under the plot) were plotted versus the concentration of Hfq. The fits of data to the Michaelis-Menten binding isotherm provided the $K_{\mathrm{d}}$ value of $0.092 \mathrm{nM}$ for ${ }^{32 P-R y b B-H f q}$, and $0.026 \mathrm{nM}$

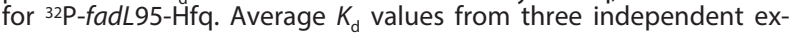
periments are provided in the text. 


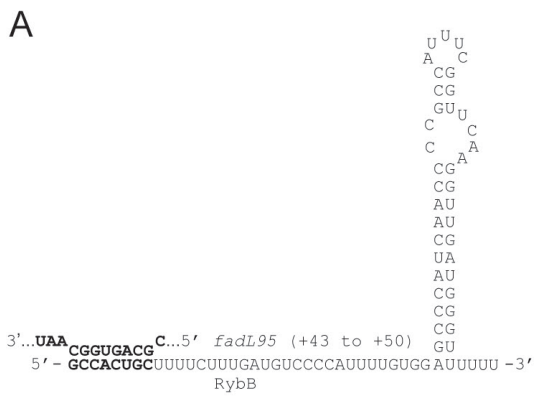

B

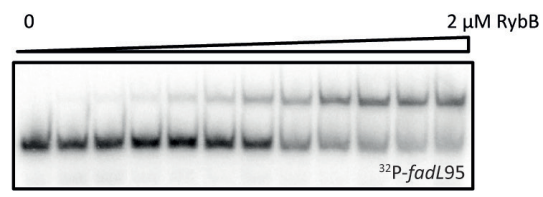

C

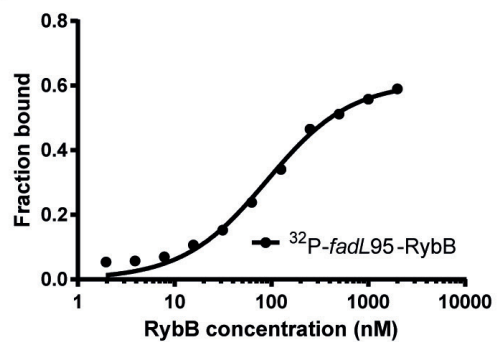

D

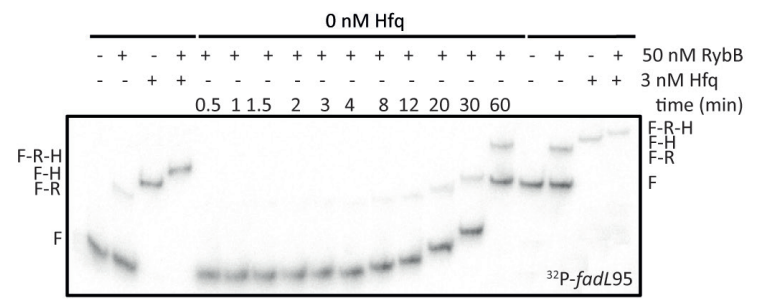

E

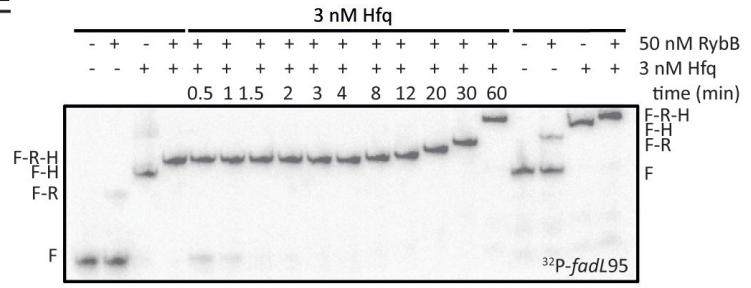

$\mathrm{F}$

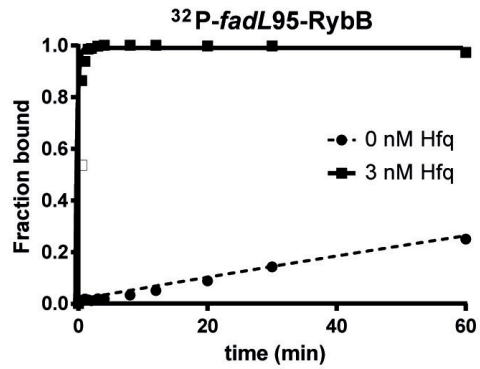

Figure 3. Hfq increases the rate of RybB annealing to the fadL95 mRNA fragment.

(A) The secondary structure of RybB sRNA showing the complementary sequence of fadL mRNA. (B) The analysis of RybB sRNA binding to 32 P-fadL95 using the native mobility shift assay. (C) The plot of ${ }^{32 P}$-fadL95 binding data from (B) versus the concentration of unlabeled RybB sRNA. The data were fit to the Michaelis-Menten equation and provided the $K_{\mathrm{d}}$ value of $89 \mathrm{nM}$. An average $K_{\mathrm{d}}$ value is provided in the text. (D) The kinetics of annealing of $1 \mathrm{nM} 32 \mathrm{P}$-fadL95 mRNA fragment to RybB (50 nM concentration) in the absence (E) or presence of $3 \mathrm{nM}$ Hfq hexamer. Free fadL95 is denoted as F, fadL95-Hfq complex as F-H, fadL95-RybB complex as F-R, fadL95-RybB-Hfq ternary complex as F-R-H. The control reaction, in which $32 \mathrm{P}$-fadL95 alone was bound to Hfq, additionally contained $2 \mathrm{nM}$ unlabeled fadL95; (F) The data from $D$ and $E$ were plotted versus time. The fitting of data to the exponential equation provided the $k_{\text {obs }}$ values of 0.0055 min $^{-1}$ and $4.0 \mathrm{~min}^{-1}$, in the absence and presence of $3 \mathrm{nM} \mathrm{Hfq}$, respectively. The average $k_{\mathrm{obs}}$ values are provided in the text.

loop for RNases. Overall, these results confirm the predicted RybB sRNA binding site in the coding sequence of fadL mRNA (Papenfort et al., 2010), and suggest that the annealing of RybB induces local rearrangements in the structure of fadL.

To test if the Hfq protein could be involved in the interactions between RybB and fadL, binding affinities of Hfq to RybB and fadL95 RNA molecules were measured using a high-throughput filter retention assay (Fig. 2). The data indicates that each of these two RNAs bound Hfq very tightly, with sub-nanomolar affinities. RybB bound Hfq with an equilibrium dissociation constant $\left(K_{\mathrm{d}}\right)$ value of $0.073 \pm 0.016 \mathrm{nM}$, while fadL95 bound Hfq with a $K_{\mathrm{d}}$ value of $0.039 \pm 0.0086 \mathrm{nM}$. The $K_{\mathrm{d}}$ value of RybB binding to $\mathrm{Hfq}$ was the same, within the error range, as the value previously reported for this interaction in a buffer containing $2 \mathrm{mM} \mathrm{Mg}{ }^{2+}$ ions (Wroblewska \& Olejniczak, 2016b). The $K_{d}$ value of Hfq binding to fadL95 was similar as previously reported for $\mathrm{Hfq}$ binding to RNA-IN mRNA (Ross et al., 2013), and ompD mRNA (Wroblewska \& Olejniczak, 2016b), and somewhat tighter than that observed for rpoS mRNA (Peng et al., 2014b), and $\mathrm{glmS}$ mRNA (Salim et al., 2012). These data suggest specific interactions of $\mathrm{Hfq}$ with both RNAs.
To test if Hfq affects the kinetics of RybB annealing to fadL95 mRNA fragment, a native gel mobility shift assay was used (Fig. 3). At first, the affinity of RybB to fadL95 was measured, which provided the $K_{\mathrm{d}}$ value of $90 \pm 13 \mathrm{nM}$ (Fig. 3A, B, C). This value is similar to that previously reported for DsrA sRNA binding to rpoS mRNA (Soper \& Woodson, 2008; Soper et al., 2011). Next, the rates of RybB annealing to fadL95 ( $\left.k_{\text {obs }}\right)$ were measured at $1 \mathrm{nM}$ concentration of ${ }^{32} \mathrm{P}$-fadL 95 and 50 $\mathrm{nM}$ unlabeled RybB in the presence or absence of 3 nM Hfq (Fig. 3D, E, F). The unbound ${ }^{32} \mathrm{P}-$ fadL95, its binary complexes with $\mathrm{Hfq}$ or RybB, and the ternary fadL95-RybB-Hfq complex migrated in the gel with distinct rates, which allowed quantifying the individual complexes. In the presence of $3 \mathrm{nM} \mathrm{Hfq}$, mostly the ternary ${ }^{32} \mathrm{P}$-fadL95-RybB-Hfq complex was formed, with a rate that was more than 300 -times faster than the rate of the binary ${ }^{32} \mathrm{P}$-fadL95-RybB complex formation in the absence of Hfq (Fig. 3D, E, F). The $k_{\text {obs }}$ value of the ternary ${ }^{32} \mathrm{P}$-fadL $95-\mathrm{RybB}-\mathrm{Hfq}$ complex formation was $3.1 \pm 0.082$, while that of the binary ${ }^{32} \mathrm{P}$-fadL95RybB complex formation in the absence of Hfq was $0.0078 \pm 0.0035$. This range of $\mathrm{Hfq}$ influence on the rate of sRNA annealing is similar to that previously observed 
for other sRNAs (Peng et al., 2014b; Wroblewska \& Olejniczak, 2016b).

\section{DISCUSSION}

The binding site of RybB sRNA in the coding sequence of fadL mRNA was predicted based on sequence analysis and confirmed using compensatory mutations in a reporter assay in vivo (Papenfort et al., 2010). Regulation of the fadL mRNA translation by RybB is among several examples of mRNAs which are regulated by sRNAs binding in the mRNA coding sequence, outside of the footprint of the initiating ribosome (Wroblewska \& Olejniczak, 2016a). Here, the direct binding of RybB to the complementary sequence in the coding region of fadL was further supported by the changes induced in the structure probing pattern of the 95-nt long fragment of fadL mRNA upon RybB annealing (Fig. 1). The decreased intensities of RNase T1 cleavages occurred in the area of predicted base-pairing of the RybB 5'-end in the fadL coding sequence, confirming its binding site (Fig 1). Moreover, the increased T1-induced cleavage, which occurred at a guanosine residue located closely upstream of the RybB binding site, was consistent with the local structural changes upon RybB binding (Fig. 1B, C, D).

The RNase T1 probing data presented here suggested that the annealing of RybB sRNA induced local structural rearrangements in the fadL mRNA coding sequence (Fig. 1B, C, D). However, as only one RNA-structure specific enzyme was used to monitor the conformational change, it is likely that the application of other structure probes, such as $\mathrm{Pb}^{2+}$ ions, could lead to a more detailed picture of this structural transition (Ciesiolka et al., 1989). In further studies, it would be also important to test the role of the structural context of a longer fadL mRNA fragment on the conformational changes in the area of RybB annealing. Local structural rearrangements induced by sRNA annealing to the mRNA coding sequence have been previously observed for other sRNAs, including MicF binding to the $h x \times \mathrm{R}$ mNA (Corcoran et al., 2012), and MicC binding to the ompD mRNA (Wroblewska \& Olejniczak, 2016b). Much larger conformational changes were observed for DsrA annealing to the 5'-UTR of rpoS mRNA, where the binding of sRNA opens up an inhibitory structure and enables the access of the ribosome to mRNA (Lease \& Woodson, 2004; Soper \& Woodson, 2008).

The Hfq protein has been shown to increase the rates of association of several sRNAs to the 5'-untranslated regions of their target mRNAs (Wagner \& Romby, 2015). However, the molecular mechanism used by Hfq to contribute to the sRNA-mRNA interaction has been explained only for the annealing of DsrA sRNA to the rpoS mRNA (Lease \& Woodson, 2004; Peng et al., 2014a; Peng et al., 2014b). Hfq rearranges the rpos mRNA structure to enable the pairing of DsrA to a complementary sequence in the mRNA (Soper \& Woodson, 2008; Soper et al., 2011). The data presented here had shown that $\mathrm{Hfq}$ increased the annealing rate of RybB sRNA to the coding sequence of fadL mRNA, and bound tightly to both of these RNAs (Fig. 2, 3). The role of Hfq in accelerating sRNA annealing to the mRNA coding sequence has been reported before for the $h x \mathrm{R}$ mRNA regulation by MicF (Corcoran et al., 2012), and the ompD mRNA regulation by $\mathrm{RybB}$, SdsR and MicC sRNAs (Wroblewska \& Olejniczak, 2016b). It was reported that Hfq strongly increased the annealing of both, RybB and
MicC, to their complementary sites located in different structural contexts in the ompD mRNA coding sequence (Wroblewska \& Olejniczak, 2016b). However, its role for RybB annealing was to overcome the energetic barriers of both RybB and ompD mRNA structures, while for MicC annealing it served only to overcome the barrier of MicC sRNA structure. The role of Hfa in sRNA annealing to the coding region depended on the $(\mathrm{ANN})_{8}$ sequence in the 5'-UTR of ompD mRNA (Wroblewska \& Olejniczak, 2016b). It is possible that the single-stranded AU-rich, (ANN) $)_{5}$ sequence in the 5'-untranslated region of fadL mRNA could be accessible for Hfq binding (Fig. 1D). However, not all Hfq binding sites are functional in the sRNA annealing. To explain the molecular mechanism used by $\mathrm{Hfq}$ to promote the RybB sRNA annealing to the fadL mRNA, further studies are needed which will involve mutants of the fadL mRNA, and Hfq variants with mutations in its RNA binding sites.

\section{Acknowledgements}

This work was supported by the KNOW RNA Research Centre in Poznań (No. 01/KNOW2/2014), National Science Centre in Poland (No. 2014/15/B/ NZ1/03330) and the Foundation for Polish Science (No. TEAM/2011-8/5) co-financed by the European Union Regional Development Fund within the framework of the Operational Program Innovative Economy.

\section{REFERENCES}

Balbontin R, Fiorini F, Figueroa-Bossi N, Casadesus J, Bossi L (2010) Recognition of heptameric seed sequence underlies multi-target regulation by RybB small RNA in Salmonella enterica. Mol Microbiol 78: 380-394. doi: 10.1111/j.1365-2958.2010.07342.x

Black PN, Said B, Ghosn CR, Beach JV, Nunn WD (1987) Purification and characterization of an outer membrane-bound protein involved in long-chain fatty acid transport in Escherichia coli. J Biol Chem 262: 1412-1419. http://www.ncbi.nlm.nih.gov/pubmed/3027089

Bouvier M, Sharma CM, Mika F, Nierhaus KH, Vogel J (2008) Small RNA binding to 5' mRNA coding region inhibits translational initiation. Mol Cell 32: 827-837. doi: 10.1016/j.molcel.2008.10.027

Chen S, Zhang A, Blyn LB, Storz G (2004) MicC, a second smallRNA regulator of Omp protein expression in Escherichia coli. J Bacteriol 186: 6689-6697. doi: 10.1128/JB.186.20.6689-6697.2004

Ciesiolka J, Wrzesinski J, Gornicki P, Podkowinski J, Krzyzosiak WJ (1989) Analysis of magnesium, europium and lead binding sites in methionine initiator and elongator tRNAs by specific metal-ioninduced cleavages. Eur J Biochem 186: 71-77. doi: 10.1111/j.14321033.1989.tb15179.x

Corcoran CP, Podkaminski D, Papenfort K, Urban JH, Hinton JC, Vogel J (2012) Superfolder GFP reporters validate diverse new mRNA targets of the classic porin regulator, MicF RNA. Mol Microbiol 84: 428-445. doi: 10.1111/j.1365-2958.2012.08031.x

Figueroa-Bossi N, Valentini M, Malleret L, Fiorini F, Bossi L (2009) Caught at its own game: regulatory small RNA inactivated by an inducible transcript mimicking its target. Genes Dev 23: 2004-2015. doi: $10.1101 / \operatorname{gad} .541609$

Guo MS, Updegrove TB, Gogol EB, Shabalina SA, Gross CA, Storz G (2014) MicL, a new sigmaE-dependent sRNA, combats envelope stress by repressing synthesis of Lpp, the major outer membrane lipoprotein. Genes Dev 28: 1620-1634. doi: 10.1101/gad.243485.114

Jorgensen MG, Thomason MK, Havelund J, Valentin-Hansen P, Storz G (2013) Dual function of the McaS small RNA in controlling biofilm formation. Genes Dev 27: 1132-1145. doi: 10.1101/ gad.214734.113

Lease RA, Woodson SA (2004) Cycling of the Sm-like protein Hfq on the DsrA small regulatory RNA. J Mol Biol 344: 1211-1223. doi: 10.1016/j.jmb.2004.10.006

Malecka EM, Strozecka J, Sobanska D, Olejniczak M (2015) Structure of bacterial regulatory RNAs determines their performance in competition for the chaperone protein Hfq. Biochemistry 54: 1157-1170. doi: $10.1021 / \mathrm{bi} 500741 \mathrm{~d}$

Milligan JF, Groebe DR, Witherell GW, Uhlenbeck OC (1987) Oligoribonucleotide synthesis using T7 RNA polymerase and synthetic DNA templates. Nucleic Acids Res 15: 8783-8798. doi: 10.1093/ nar/15.21.8783 
Nunn WD, Simons RW (1978) Transport of long-chain fatty acids by Escherichia coli: mapping and characterization of mutants in the fadL gene. Proc Natl Acad Sci U S A 75: 3377-3381. http://www.ncbi. nlm.nih.gov/pubmed/356053

Olejniczak M (2011) Despite similar binding to the Hfq protein regulatory RNAs widely differ in their competition performance. Biochemistry 50: 4427-4440. doi: 10.1021/bi102043f

Panja S, Santiago-Frangos A, Schu DJ, Gottesman S, Woodson SA (2015) Acidic residues in the Hfq chaperone increase the selectivity of sRNA binding and annealing. J Mol Biol 427: 3491-3500. doi: 10.1016/j.jmb.2015.07.010

Panja S, Schu DJ, Woodson SA (2013) Conserved arginines on the rim of Hfq catalyze base pair formation and exchange. Nucleic Acids Res 41: 7536-7546. doi: 10.1093/nar/gkt521

Papenfort K, Bouvier M, Mika F, Sharma CM, Vogel J (2010) Evidence for an autonomous 5' target recognition domain in an Hfqassociated small RNA. Proc Natl Acad Sci U S A 107: 20435-20440. doi: 10.1073 /pnas.1009784107

Papenfort K, Vogel J (2010) Regulatory RNA in bacterial pathogens. Cell Host Microbe 8: 116-127. 10.1016/j.chom.2010.06.008

Parker A, Gottesman S (2016) Small RNA Regulation of TolC, the outer membrane component of bacterial multidrug transporters. $J$ Bacteriol 198: 1101-1113. doi: 10.1128/JB.00971-15

Peng Y, Curtis JE, Fang X, Woodson SA (2014a) Structural model of an mRNA in complex with the bacterial chaperone Hfq. Proc Nat Acad Sci U S A 111: 17134-17139. doi: 10.1073/pnas.1410114111

Peng Y, Soper TJ, Woodson SA (2014b) Positional effects of AAN motifs in rpoS regulation by sRNAs and Hfq. J Mol Biol 426: 275285. doi: 10.1016/j.jmb.2013.08.026

Pfeiffer V, Papenfort K, Lucchini S, Hinton JC, Vogel J (2009) Coding sequence targeting by MicC RNA reveals bacterial mRNA silencing downstream of translational initiation. Nat Struct Mol Biol 16: 840846. doi: 10.1038/nsmb.1631

Reuter JS, Mathews DH (2010) RNAstructure: software for RNA secondary structure prediction and analysis. BMC Bioinformatics 11: 129. doi: 10.1186/1471-2105-11-129

Ross JA, Ellis MJ, Hossain S, Haniford DB (2013) Hfq restructures RNA-IN and RNA-OUT and facilitates antisense pairing in the Tn10/IS10 system. RNA 19: 670-684. doi: 10.1261/rna.037747.112

Salim NN, Faner MA, Philip JA, Feig AL (2012) Requirement of upstream Hfq-binding (ARN) $x$ elements in glmS and the Hfq C-ter- minal region for GlmS upregulation by sRNAs GlmZ and GlmY. Nucleic Acids Res 40: 8021-8032. doi: 10.1093/nar/gks392

Santiago-Frangos A, Kavita K, Schu DJ, Gottesman S, Woodson SA (2016) C-terminal domain of the RNA chaperone Hfq drives sRNA competition and release of target RNA. Proc Natl Acad Sci USA. doi: $10.1073 /$ pnas.1613053113

Schu DJ, Zhang A, Gottesman S, Storz G (2015) Alternative HfqsRNA interaction modes dictate alternative mRNA recognition. EMBO J 34: 2557-2573. doi: 10.15252/embj.201591569

Soper TJ, Doxzen K, Woodson SA (2011) Major role for mRNA binding and restructuring in sRNA recruitment by Hfq. RNA 17: 1544 1550. doi: $10.1261 / \mathrm{rna} .2767211$

Soper TJ, Woodson SA (2008) The rpoS mRNA leader recruits Hfq to facilitate annealing with DsrA sRNA. RNA 14: 1907-1917. doi: $10.1261 /$ rna.1110608

Tree JJ, Granneman S, McAteer SP, Tollervey D, Gally DL (2014) Identification of bacteriophage-encoded anti-sRNAs in pathogenic Escherichia coli. Mol Cell 55: 199-213. doi: 10.1016/j.molcel.2014.05.006

Updegrove TB, Zhang A, Storz G (2016) Hfq: the flexible RNA matchmaker. Curr Opin Microbiol 30: 133-138. doi: 10.1016/j. mib.2016.02.003

Wagner EG, Romby P (2015) Small RNAs in bacteria and archaea: who they are, what they do, and how they do it. Adv Genet 90: 133208. doi: 10.1016/bs.adgen.2015.05.001

Waters LS, Storz G (2009) Regulatory RNAs in bacteria. Cell 136: 615628. doi: 10.1016/j.cell.2009.01.043

Wroblewska Z, Olejniczak M (2016a) Contributions of Hfq protein to translation regulation by small noncoding RNAs binding to the mRNA coding sequence Acta Biochim Pol 63: 840-707. https://doi. org/10.18388/abp.2016_1362

Wroblewska Z, Olejniczak M (2016b) Hfq assists small RNAs in binding to the coding sequence of ompD $\mathrm{mRNA}$ and in rearranging its structure. RNA 22: 979-994. doi: 10.1261/rna.055251.115

Zhang A, Schu DJ, Tjaden BC, Storz G, Gottesman S (2013) Mutations in interaction surfaces differentially impact $E$. coli $\mathrm{Hfq}$ association with small RNAs and their mRNA targets. $J$ Mol Biol 425: 3678-3697. doi: 10.1016/j.jmb.2013.01.006

Zheng A, Panja S, Woodson SA (2016) Arginine patch predicts the RNA annealing activity of $\mathrm{Hfq}$ from gram-negative and grampositive bacteria. J Mol Biol 428: 2259-2264. doi: 10.1016/j. jmb.2016.03.027 